\title{
Orthoscopic, long-focal-depth integral imaging by hybrid method
}

\author{
Bahram Javidi, Raúl Martínez-Cuenca ${ }^{+}$, Genaro Saavedra ${ }^{+}$, and Manuel Martínez-Corral ${ }^{+}$ \\ Electrical and Computer Engineering Dept, University of Connecticut, Storrs, CT 06269-1157 \\ ${ }^{+}$Department of Optics, University of Valencia, E-46100 Burjassot, Spain.
}

\begin{abstract}
Integral imaging systems are imaging devices that provide 3D images of 3D objects. When integral imaging systems work in their standard configuration they provide reconstructed images that are pseudoscopic, distorted and with very poor depth of field. Along the last four years our group has been working in the search of solutions for these drawbacks. Here we present hybrid technique which by means of optical method and digital processing allows the reconstruction of orthoscopic, undistorted, long-focal-depth integral images. Simulated and real imaging experiments are presented to support our proposal.
\end{abstract}

Key words: Integral imaging, 3D imaging display, digital processing

\section{INTRODUCTION}

One of the challenges of information society is the development, and subsequent broad implantation, of technologies for acquisition and display of three-dimensional (3D) pictures and movies. The search the optimum 3D imaging/display technique has been the aim of research efforts for long time [1]. However, only in the past few years has the technology reached the level required for the realization of such kind of systems. Among the existing 3D imaging techniques the one known as integral imaging (InI) is especially appreciated because it has the ability of providing real or virtual autostereoscopic intensity images with full parallax, without the help of any special glasses. In an InI system the perspective information of a 3D scene is stored in a collection of 2D images, called elemental images, usually arranged in rectangular grid. The main advantage of an InI monitor is the fact that one observer can see different perspectives of a 3D scene by simply varying the head position [2]. InI was first proposed by Lipmann about one century ago [3]. The interest in InI resurrected recently because of its application to 3D TV [4]. Based on an attractive concept, the first designs of InI systems suffered from many troubles. From that time, much research has been addressed to tackle, satisfactorily in many cases, such troubles. Let us remark for example, the efforts done for improving the lateral resolution [57], for enhancing the depth of field [8,11], for pseudoscopic to orthoscopic conversion, or for minimizing the elementalimage overlap $[12,13]$. InI system have shown to be useful not only for pure 3D imaging but also for other applications like object recognition [14] or the mapping of 3D polarization distributions [15].

Although our group has been working in the field of InI only for the last four years it can be recognized that we have already made some interesting contributions, which help to deal satisfactorily with some of the main challenges of 3D imaging recording/display system. This paper is devoted to review these achievements. Concerning the limitation in depth of field, it is relevant that in the pickup stage the limitation is imposed by pixilation and diffraction. In the display stage the limitation is imposed by a phenomenon that is inherent to InI systems and to which we have given the name of facet braiding. Other challenges we have dealt satisfactorily are the acquisition of non-shifted non-overlapped elemental images and the pseudoscopic to orthoscopic conversion. Finally we present a method for three-dimensional information extraction from elemental image collection.

\section{PRINCIPLES OF INTEGRAL IMAGING}

In Lipmann scheme, photography of a self-luminous 3D scene is taken through a pinhole array. This pickup setup provides with a set of micro-images, named hereafter to as the elemental images, each having information of a different perspective of the object. In the display stage properly processed film is placed in front of the same pinhole array and back illuminated so that each elemental image acts as a spatially incoherent object. Note that this setup allows the reconstruction of a real, but depth reversed image of the 3D scene.

Three-Dimensional TV, Video, and Display V, edited by Bahram Javidi, Fumio Okano, Jung-Young Son Proc. of SPIE Vol. 6392, 639203, (2006) · 0277-786X/06/\$15 · doi: 10.1117/12.686659 

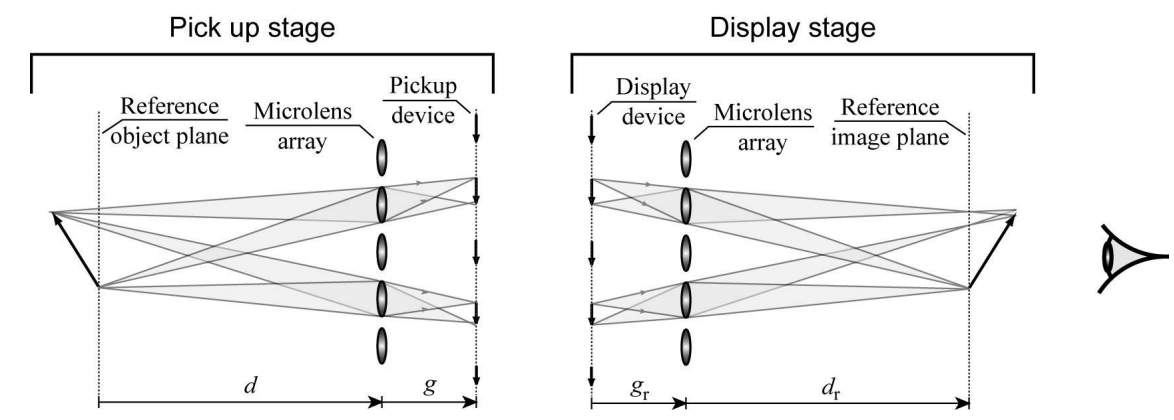

Figure 1.- Scheme of an integral imaging system

The major drawback of the scheme proposed by Lipmann is the poor light efficiency, the second problem is that the film processing inherent to a photographic process is very slow and does not match the current requirements of 3D imaging. A solution of these problems is to substitute the pinhole array by a microlens array and the photographic film by an electronic matricial sensor like a CCD or a CMOS. As shown in Fig. 1, now the elemental images are formed in the image plane of the microlenses. However, now an important problem appears: the limitation in DOF. Due to the imaging properties of the lenses, now only one plane of the object space is in focus. Then light proceeding from other planes does not focus onto the CCD. The same problem happens in the display stage. Only points within the object plane are reconstructed sharply. Other parts of the 3D scene are increasingly blurred. Besides, this blurring effect is more significant when the pixilation of the CCD (or the LCD in the display stage) is taken into account. Consequently, this new realization of the integral imaging process gives rise to much more luminous reconstructed images, but with low resolution even in case of the in-focus plane.

\section{DEPTH OF FIELD}

One of the challenges that InI is facing is the limited depth of field. In a typical scene objects exist at different depth positions. Since only a single object plane is used to capture the images, it is not possible for all objects to be in focus. Then blurred images of out-of-focus objects are obtained. Although the depth of field (DOF) of InI systems is influenced by many parameters (related with both the capture and the display systems), it is apparent that to display a clear integral image of an axially elongated 3D object it is essential to capture sharp 2D elemental images of it. For this reason, the bottleneck of the DOF in integral imaging is the limited depth of field of the microlens array used in the pickup stage. One could overcome this problem by reducing the numerical aperture (NA) of the lenses. However, such a reduction would produce, as a collateral effect, an important worsening of lateral resolution of capture stage, and therefore of spatial resolution of the overall integral imaging system.

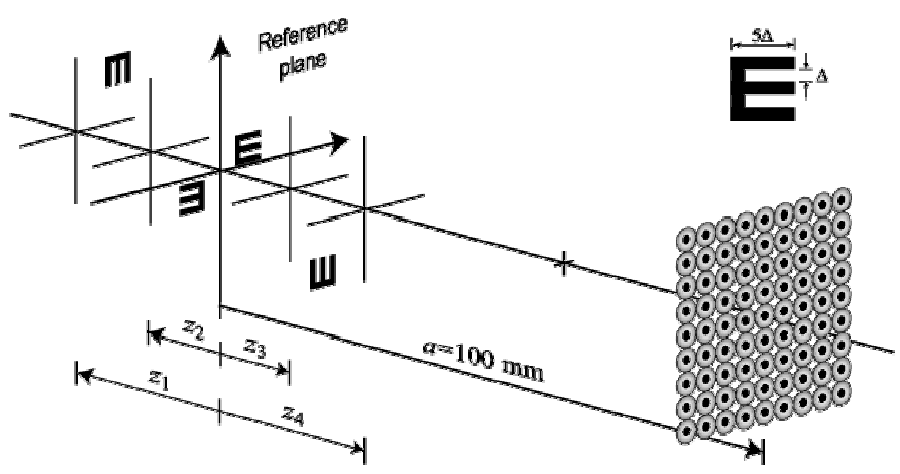

Figure 2. Simulated pickup experiment in which the annular aperture is inserted as entrance pupil of the microlenses.

To overcome this problem, our proposal is based on the use of binary amplitude modulation technique. As well known, an imaging system whose aperture stop is apodized with an annular aperture, produce a diffractive spot that, when compared with the one produced by the usual circular aperture, is narrower in the lateral direction and much larger in the axial direction. This allows the production of images with higher depth of field and no deterioration of lateral resolution. The only real problem of this technique, when applied to InI systems, is how to insert the grid of annular apertures 
in front of the array of microlenses. We have found however a very simple, cheap optical technique to resolve such problem. Our solution consists on the use of a proper relay system that allows the projection of a grid of micro-images of a unique annular hard aperture, onto the front focal plane of any of the microlenses (see Fig. 2).

As we can see in Fig. 3, the use of the annular apertures allows the significant improvement of the depth of field of pickup system, allowing then the capture of sharp images for a large range of axial positions.
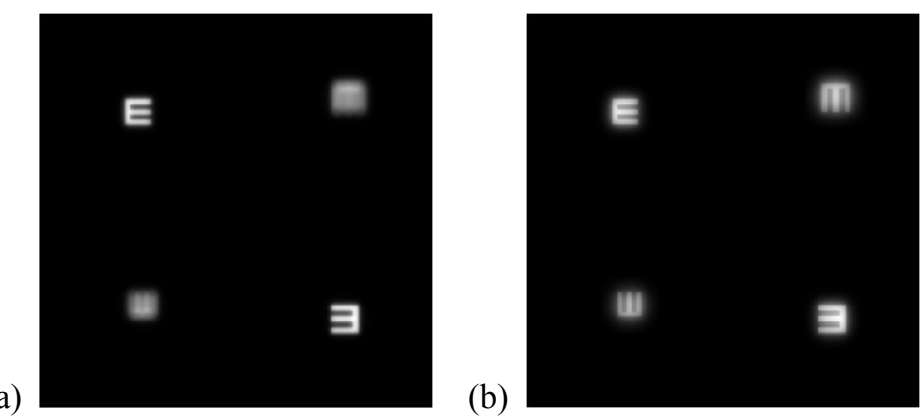

Figure 3 .Elemental images obtained with: (a) The array of microlenses with circular aperture; (b) The array of microlenses with annular aperture.

The main limitation in DOF during the reconstruction comes from the braiding of visual facets. As consequence of the facet-braiding effect, the effective DOF of the reconstructed images importantly decreases, even in the ideal case in which the pickup was realized with infinite DOF. To get an intuitive understanding the nature of the facet-braiding phenomenon, we start by drawing an scheme of the pickup stage of an InI system. As shown in Fig. 4, an object placed at the reference plane produces a collection of sharp elemental images. In case of out-of focus objects the scale, the light proceeding from out-of-focus points does not focus sharply onto the sensor, so that the elemental images are blurred. We will assume however in the forthcoming analysis that techniques for DOF enlargement are applied, so that a large range of axial object positions are recorded sharply.

(a)

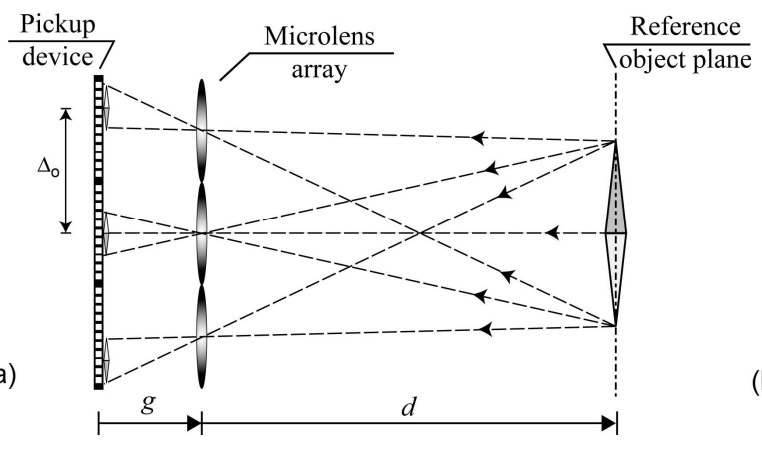

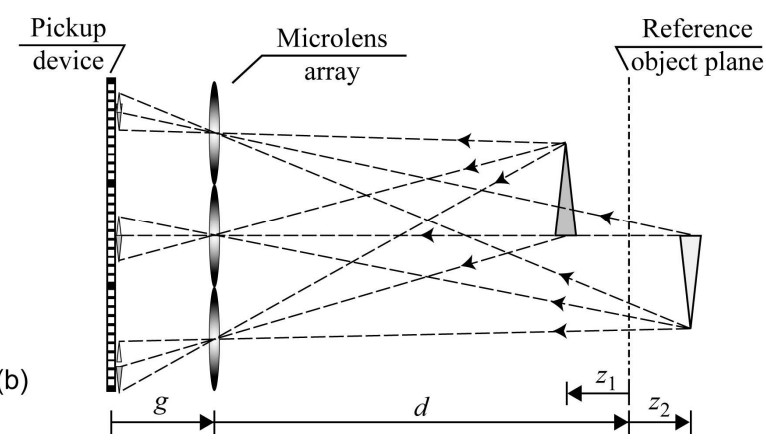

Fig. 4 Scheme of the pickup stage of an InI system: (a) Elemental images of an in-focus object; (b) Elemental images of out-of-focus scenes.

Next in Fig. 5, we schematize the reconstruction and visualization process. This process is the results of three phenomena: a geometrical projection, an image formation process and, finally, the arrangement of the collection of elemental fields of view (FOVs), also know as visual facets. In case of in-focus objects a full consonance between the three phenomena occurs. (i) The rays proceeding from the elemental images, and passing through the center of the microlenses, intersect at the reference plane so that the image is reconstructed with the same position and size as the object; (ii) The pickup-device plane and the reference image plane are conjugate through the microlenses. Then, the images generated by the elemental images through the corresponding microlenses, coincide in size and position; (iii) When the observer places the eye in front of the lenslets array and looks through it, he/she sees a different portion of the reconstructed image through each microlens. In the case of out-of-focus objects the consonance is broken. (i) The image is reconstructed, again, with the same position and size as the object. (ii) The pickup-device plane and the reference image plane are still conjugate through the microlenses. Then, the image generated by any elemental image through the corresponding microlens does not appear in the same position as the reconstructed image, but in the reference plane. Al- 
though all these images have the same size, they appear centered at different positions. (iii) In the observation stage, what the observer sees through each microlens is a portion of the image provided by such the microlens. But now the images provided by the microlenses are displaced so that the visual facets are arranged in a cracked relief. The cracking follows a braiding path. For scenes placed between the MLA and the reference plane, the images provided by the microlenses suffer an inverse displacement. Inverse displacement means that upper lenses provide lower images. For scenes at the reference plane there is not displacement. For scenes further than the reference plane the displacement is direct.
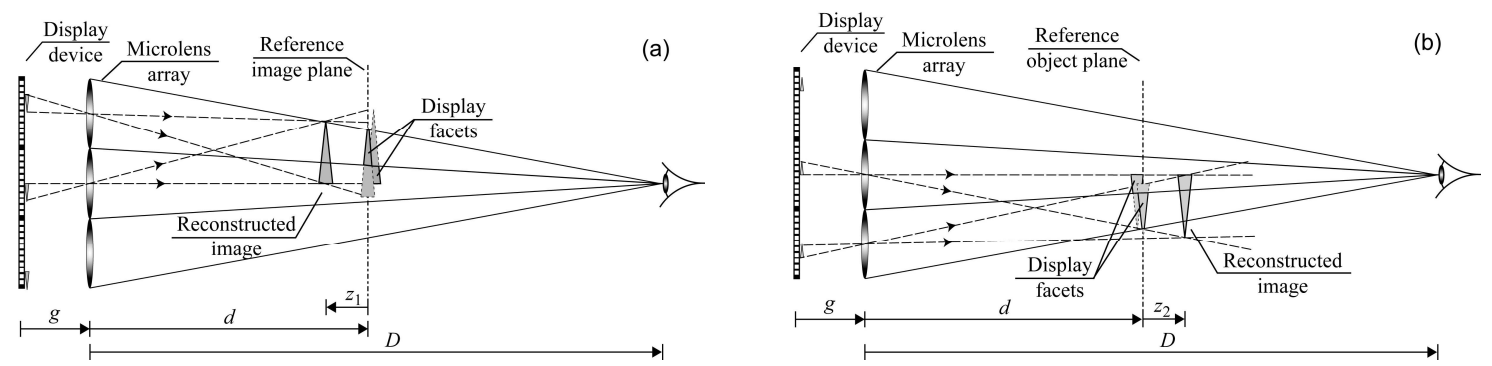

Fig. 5 Display stage of an InI system. Whatever the object position, the image is reconstructed with the same position and size as the object. However, what the observer sees are portions of the images provided by the microlenses. (a) The images suffer an inverse displacement; (b) The images suffer a direct displacement.

To illustrate the importance of the facet-braiding phenomenon we performed an experiment in which we obtained the elemental images of a 3D scene consisting on three capital letters, namely R, G and B, each printed on a different plate and located at distances $40 \mathrm{~mm}, 100 \mathrm{~mm}$ and $150 \mathrm{~mm}$ from the MLA, respectively. The InI system was arranged so that the reference plane was set at $d=100 \mathrm{~mm}$. Although the $\mathrm{R}$ and $\mathrm{G}$ were clearly out-of-focus scenes, the pickup was arranged so that a high DOF was obtained, and therefore they were recorded sharply (see Fig. 6).
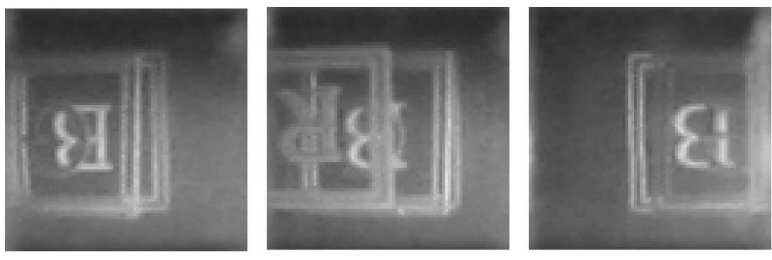

Fig. 6 Three elements of the same row of the elemental-image collection. Note that out-of-focus scenes were recorded sharply.

We simulated the display stage by computer processing. In Fig. 7(a) we show a view of the reconstructed image. It is clear from the figure that only the in-focus letter (letter $\mathrm{G}$ ) is observed without distortions. A proof of it is that the dark band in the square frame surrounding the letter is clearly resolved. In the other letters case the facet braiding produce a distortion that impoverishes the quality of both the letters and the square frame.
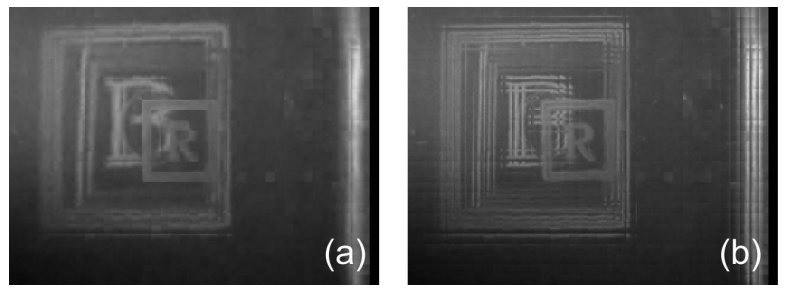

Fig. 7 Views of reconstructed 3D scene. (a) The reference plane was set, as in the pickup, at $d=100 \mathrm{~mm}$; (b) The reference plane for reconstruction was set at $\mathrm{d}=40 \mathrm{~mm}$.

To solve this problem we propose the use of dynamic focusing technique during the display stage. So we have simulated the reconstruction stage for a new value of the microlenses focal length, namely $f_{1}=3.12 \mathrm{~mm}$. This allows us to displace the reference plane to $d_{1}=40 \mathrm{~mm}$. In Fig. 7(b) we show the view corresponding to the new reconstruction 
stage. Note how the dynamic focusing permits the selection of the plane free of facet-braiding effect. Now the square frame surrounding the letter $\mathrm{R}$ is resolved. In planes far from this plane the facet braiding is enhanced and typical banding appears.

\section{PSEUDOSCOPIC TO ORTHOSCOPIC CONVERSION}

Let us consider the standard configuration of an InI system, as shown in Fig. 1. In the pickup stage the system is adjusted so that a representative plane of the object neighborhood, referred here as the reference object plane, and the CCD plane are conjugated through the microlenses. Distances $d$ and $g$ are, then, related through the lens law, $1 / d+1 / g=1 / f, f$ being the lenslets focal length. Then, a collection of elemental 2D images, each with a different perspective of the 3D object, are generated onto the pickup device. In the reconstruction process the recorded elemental images are displayed by an optical device (like a LCD) in front of the other microlens array. The LCD and the microlens array are adjusted so that the gap, $g_{\mathrm{r}}$, is the same as the pickup gap, $g$. As shown in the figure this architecture permits the reconstruction of a real but pseudoscopic image at a distance $d_{\mathrm{r}}=d$.

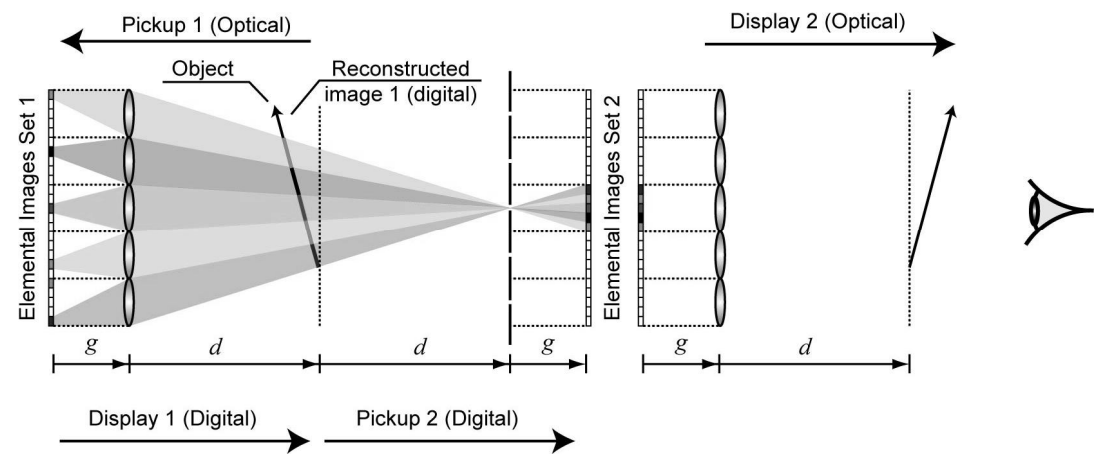

Fig. 8 Scheme of the PO digital conversion method.

Among the techniques proposed for the pseudoscopic to orthoscopic (PO) conversion $[4,17-22]$ the only one that satisfies the requirement of providing real, orthoscopic 3D images is the one proposed by Ives [16]. However, the practical application of this technique is not very advantageous because the two-step recording/displaying technique produces a strong degradation in the image. What we suggest here is to make use of Ives PO concept but, in order to avoid the disadvantages of the two-step process, we propose to perform the intermediate stage (Display 1 + Pickup 2) by digital procedure. On the basis of the scheme shown in Fig. 8, we will explain the PO digital procedure for the 1D case. The extension to the 2D case is straightforward. Since we assume that the optically-acquired set of elemental images (Set 1) have a pixilated structure, it is clear that the conversion to the second set of elemental images (Set 2) will simply consist in a pixel mapping. Then, to avoid typical aliasing problems in the pixels mapping, it is necessary to assume that the following relations are satisfied: $N=m M$ and $M=2 d / g$, where $N$ stands for the number of pixels per elemental cell, $M$ is the number of elemental images, and $m$ is a positive integer number. Since there is no loss of energy in the digital pickup, we perform the digital Pickup 2 through a virtual pinhole array. This simplifies the process.

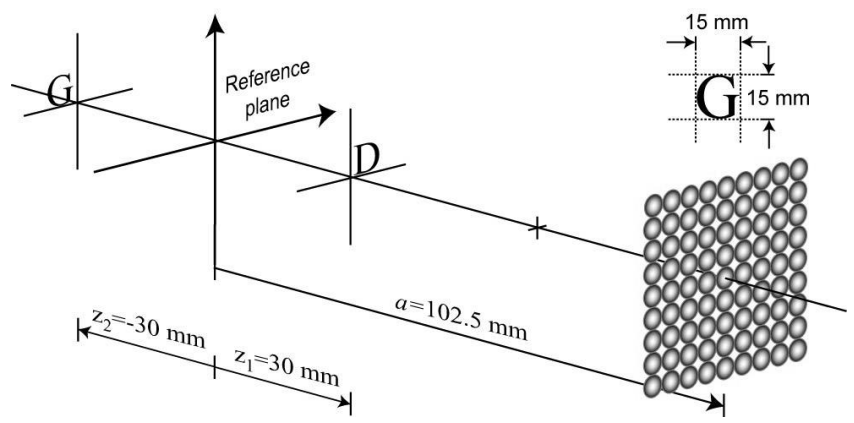

Fig. 9. Scheme, not to scale, of the pickup numerical experiment. The CCD is adjusted so that the reference object plane is at $102.5 \mathrm{~mm}$. The letters are separated by $60 \mathrm{~mm}$ in depth. 
To illustrate our approach we have simulated the experiment shown in Fig. 9. In Fig. 10 we show both, the original and the mapped collection of elemental images. Finally in Fig. 13 we simulated the observation by an observer that looks towards the central microlens, and displaces the eye horizontally from $x=-25 \mathrm{~mm}$ to $\mathrm{x}=-25 \mathrm{~mm}$.
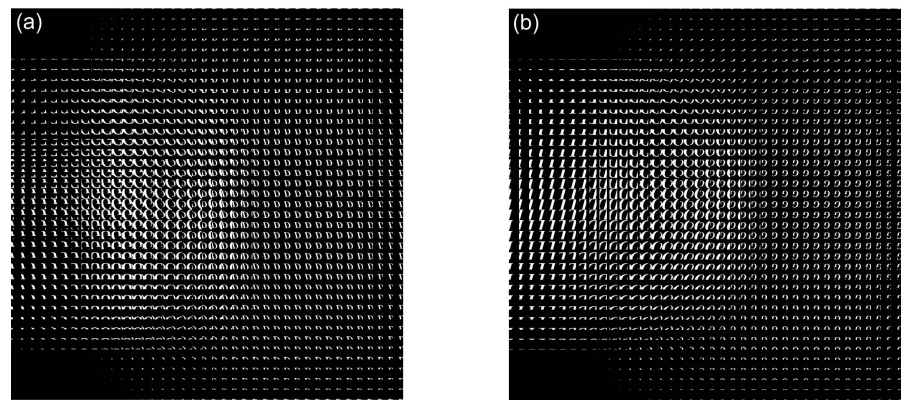

Fig. 10 (a) Collection of $39 \times 39$ elemental images obtained with the setup of previous figure; (b) Elemental images obtained after applying the smart pixel mapping.

(a)
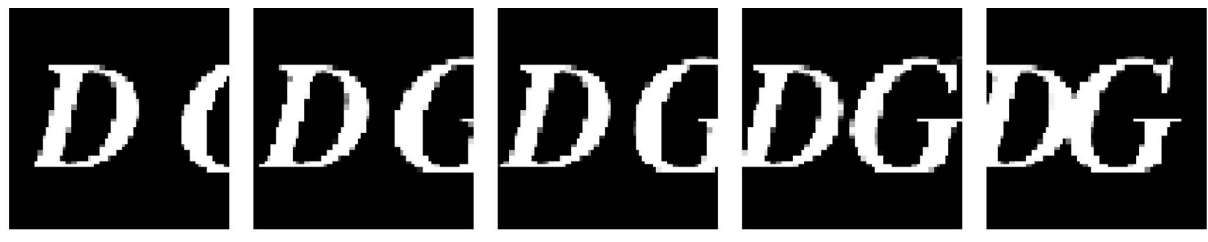

(b)
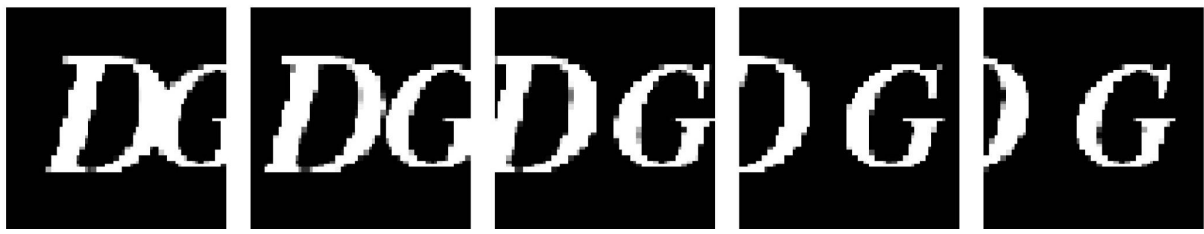

Fig. 11 (a) Real, pseudoscopic image calculated from the elemental images in Fig. 11(a); (b) Real orthoscopic image calculated from the elemental images in Fig. 11(b). In both reconstructions we assume that the observer is placed at $500 \mathrm{~mm}$ from the microlenses, and he/she laterally displaces the eye from left to right The eye displacement is from $\mathrm{x}=-25 \mathrm{~mm}$ to $\mathrm{x}=+25 \mathrm{~mm}$.

\section{CORRECTION OF MICROIMAGES OVERLAP AND SHIFT}

As shown in Fig. 1, in the pickup stage a collection of elemental images, each with different perspective of the 3D scene, is generated onto the pickup device. Note however that this schematic geometry cannot be used for real pickup. The problem comes from the fact that when capturing large scenes, the different microimages are much larger than the corresponding elemental cells, giving rise to a strong overlapping between microimages. In this case, any elemental cell receives light from many microlenses and therefore no useful information can be extracted from them. In order to obtain high-quality 3D reconstructions a pickup architecture that provides a collection of non-overlapped microimages whose size and position match the elemental-cell grid is needed. To obtain this one should realize that: (a) the center of each micro-vignette is located just at the intersection of the aerial-images plane and the line joining the center of the microlens with the center of the relay-system entrance pupil (EP); and (b) the extension of each micro-vignette is determined by joining the center of the relay-system EP with the borders of the corresponding microlens. Therefore, to allow the micro-vignettes collection to match the elemental-cells grid, a relay system where the EP is placed at infinity is needed. Besides, the conjugate of this EP through the different microlenses, which will be called micro-EPs, must be small enough to avoid the overlapping effect. In other words, the relay system must be telecentric in its object space [23] and with an aperture stop such that the corresponding micro-EPs are small enough to minimize the overlapping. However, one must take into account that when the micro-EPs are too small diffraction effects do appear, which im- 
poverishes the lateral resolution of the system. Thus, the proper selection of the aperture stop diameter should be the result of the trade-off between the overlapping effect and the diffraction limit.

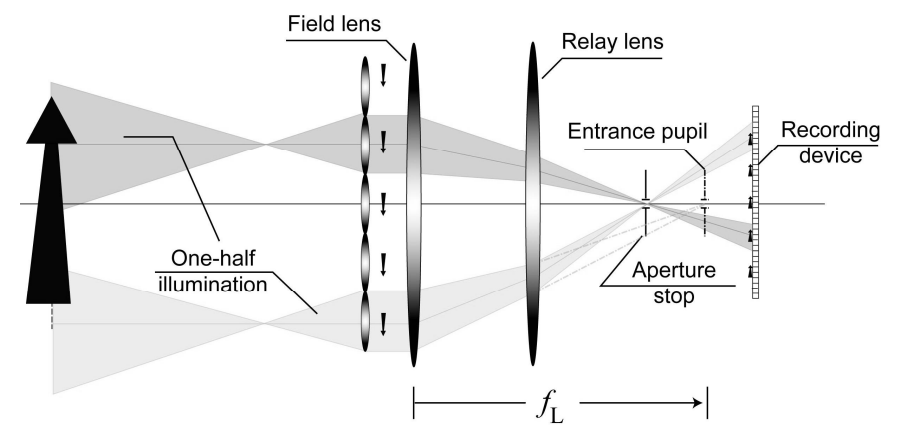

Fig. 12 Scheme of proposed pickup stage. The telecentric relay system allows the micro-vignettes to match the elemental-cells grid.

On the basis of the above reasoning, we propose a new architecture for the pickup stage. Our proposal is schematized in Fig. 12. The relay system is composed of a high-diameter converging lens, the field lens, and a macro objective, which as in Fig. 1 is schematized by the relay lens and the aperture stop. The macro and the field lens should be arranged so that the relay system is telecentric. As shown in the figure, this system permits the capture of a collection of microimages that match the elemental-cell grid. In other words, this setup permits the acquisition, by optical means, of the correct elemental-image collection.

\section{CONCLUSIONS}

We have performed a review of the achievements of our group in the context of InI systems. Specifically, we show an optical technique for improvement of the depth of field in the pickup stage, which allows the recording of sharp elemental images for a long range of axial positions. Besides, we present a technique that minimizes the overlap, also known as interference, between elemental images by means of an adequate arrangement of the pickup relay system. Finally we present a image processing technique that globally rearrange the pixels of the integral-image collection, so that a real orthoscopic image reconstruction is possible.

\section{ACKNOWLEDGEMENTS}

This work has been funded in part by the Plan Nacional I+D+I (grant DPI2003-4698), Ministerio de Ciencia y Tecnología, Spain. R. Martínez-Cuenca acknowledges funding from the Universitat de València (Cinc Segles grant). We also acknowledge the support from the Generalitat Valenciana (grant GV06/219).

\section{REFERENCES}

1. T. Okoshi, "Three-dimensional displays," Proc. IEEE 68, 548-564 (1980).

2. F. Okano, "Real-time integral imaging based on extremely high resolution video system," Proc. IEEE 94, 490-501 (2006).

3. M. G. Lippmann, "Epreuves reversibles donnant la sensation du relief," Journal of Physics (Paris) 7, 821-825 (1908).

4. F. Okano, H. Hoshino, J. Arai and I. Yayuma, "Real time pickup method for a three-dimensional image based on integral photography," Appl. Opt. 36, 1598-1603 (1997).

5. A. Stern and B. Javidi, "Three-dimensional image sensing and reconstruction with time-division computational integral imaging," Appl. Opt. 42, 7036-7042 (2003).

6. S. Manolache, A. Aggoun, M. McCormick and N. Davies, "Analytical model of a three-dimensional integral image recording system that uses circular and hexagonal-based spherical surface microlenses," J. Opt. Soc. Am. A 18, 1814-1821 (2001). 
7. J.-S. Jang and B. Javidi, "Improved viewing resolution of three-dimensional integral imaging by use of nonstationary micro-optics," Opt. Lett. 27, 324-326 (2002).

8. R. Martínez-Cuenca, G. Saavedra, M. Martínez-Corral and B. Javidi, "Enhanced depth of field integral imaging with sensor resolution constraints," Opt. Express 12, 5237-5242 (2004).

9. M. Martínez-Corral, B. Javidi, R. Martínez-Cuenca and G. Saavedra, "Integral imaging with improved depth of field by use of amplitude-modulated microlens array," Appl. Opt. 45, 5806-5813 (2004).

10. R. Martínez-Cuenca, G. Saavedra, M. Martínez-Corral and B. Javidi, "Extended depth-of-field 3-D display and visualization by combination of amplitude-modulated microlenses and deconvolution tools," J. Disp. Technol. 1, 321-327 (2005).

11. B. Lee, S.-W. Min and B. Javidi, "Theoretical analysis for three-dimensional integral imaging with double devices," Appl. Opt. 41, 4856-4865 (2002).

12. N. Davies, M. McCormick and L. Yang, "Three-dimensional imaging systems: a new development," Appl. Opt. 27, 4520-4528 (1988).

13. M. Martínez-Corral, B. Javidi, R. Martínez-Cuenca and G. Saavedra, "Formation of real, orthoscopic integral images by smart pixel mapping," Opt. Express 13, 9175-9180 (2005).

14. Y. Frauel, O. Matoba, E. Tajahuerce and B. Javidi, "Comparison of passive ranging integral imaging and active imaging digital holography for 3D object recognition," Appl. Opt. 43, 452-462 (2004).

15. O. Matoba and B. Javidi, "Three-dimensional polarimetric integral imaging," Opt. Lett. 29, 2375-2377 (2004).

16. H. E. Ives, "Optical properties of a Lippmann lenticulated sheet," J. Opt. Soc. Am. 21, 171-176 (1931).

17. N. Davies, M. McCormick, and L. Yang, "Three-dimensional imaging systems: a new development," Appl. Opt. 27, 4520-4528 (1988).

18. J.-H. Park, S.-W. Min, S. Jung, and B. Lee, "Analysis of viewing parameters for two display methods based on integral photography," Appl. Opt. 40, 5217-5232 (2001)

19. M. Martínez-Corral, B. Javidi, R. Martínez-Cuenca, and G. Saavedra, "Mutifacet structure of observed reconstructed integral images," J. Opt. Soc. Am. A 22, 597-603 (2005).

20. J-S. Jang and B. Javidi, "Two-step integral imaging for orthoscopic three-dimensional imaging with improved viewing resolution," Opt. Eng. 41, 2568-2571 (2002).

21. J-S. Jang and B. Javidi, "Three-dimensional projection integral imaging using micro-convex-mirror arrays," Opt. Express 12, 1077-1083 (2004).

22. J.-S. Jang and B. Javidi, "Formation of orthoscopic three-dimensional real images in direct pickup one-step integral imaging," Opt. Eng. 42, 1869-1870 (2003).

23. M. Martínez-Corral, M. T. Caballero, L. Muñoz-Escrivá and P. Andrés, "Focal shift formula in apodized nontelecentric focusing systems," Opt. Lett. 26, 1501-1503 (2001). 